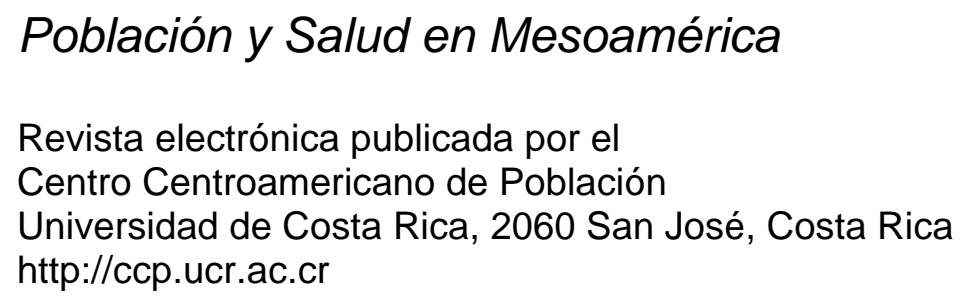

Población y Salud en Mesoamérica

Revista Electrónica

Volumen 3, número 2, artículo 3

Enero - junio, 2006

Publicado 1 de enero, 2006

http://ccp.ucr.ac.cr/revista/

\title{
Construcción de un indicador para medir competencias básicas para la vida
}

\section{Andrea Collado Chaves}

(C) 2006 Centro Centroamericano de Población 


\title{
Construcción de un indicador para medir competencias básicas para la vida
}

\author{
Andrea Collado Chaves ${ }^{1}$
}

\section{RESUMEN}

La necesidad de construir un indicador para cuantificar el nivel de Competencias Básicas para la Vida (CBV), nace con la evaluación los resultados de un proyecto dirigido a población joven. El propósito de ese proyecto es construir capital humano y empleabilidad en hombres y mujeres con edades entre 13 y 18 años, desertores o en riesgo de desertar del sistema educativo, sin empleo formal y que viven en ambientes de pobreza y exclusión. Una de las dimensiones de empleabilidad son las Competencias Básicas para la Vida. La dimensión de competencias básicas para la vida se refiere a aspectos de personalidad asociados con el desempeño del individuo en sus relaciones consigo mismo y con los demás. Los datos provienen de una investigación cuyo propósito consiste en evaluar los resultados de una intervención en población joven de comunidades pobres y excluidas. La construcción del indicador uso técnicas psicométricas para la validación de las distintas subdimensiones que pertenecen al concepto de competencias básicas para la vida. Según el marco conceptual este indicador está compuesto por seis dimensiones, la validación empírica hecha en este artículo incluye solamente cuatro de ellas, todas de carácter actitudinal. El indicador posee algunas ventajas en términos de la capacidad de discriminación debido a que su distribución tiende a ser normal. Sin embargo se ve afectaba por valores extremos a la izquierda del promedio y valores modales a una desviación estándar (positiva) del promedio. Estos resultados serán útiles para discusiones futuras sobre los puntos de corte (baremos), el comportamiento del indicador cuando se correlaciona con deseabilidad social y la integración de la dimensión de Competencias Básicas para la Vida en el indicador de empleabilidad.

\section{ABSTRACT}

This papers use psychometrics techniques to create an indicator for quantifying the level of Basic Life Capabilities that refers to personality aspects associated with the individual' behavior and the way young people relates with others. According to the conceptual framework the Basic Life Capabilities indicator has six dimensions: interpersonal skills, attitudes toward gender, capacity to work under pressure, among others; the empiric validation and the reliability tests include four of them. The indicator is a dimension of employability concept that will be useful for an evaluation on a project for young population. The purpose of this project is to build human capital and employability in young men and women age 13 to 18 , which dropped out or are in risk of dropping out school. These young people also do not have access to a formal job and live in poor and excluded communities. Data comes from the first wage of the result evaluation. Results show the indicator accomplishes most of the statistical properties, do not covariate with gender or residence, but improves the scores while young are getting older and young are attending school. This evidence will be useful for creating the employability index and further discussions.

\footnotetext{
${ }^{1}$ Master en Población y Salud. Investigadora del Centro Centroamericano de Población de la Universidad de Costa Rica. Profesora, Escuela de Estadística y Sistema de Estudios de Posgrado Docente, Universidad de Costa Rica. acollado@ccp.ucr.ac.cr
} 


\section{INTRODUCCIÓN}

La necesidad de construir un indicador para cuantificar el nivel de Competencias Básicas para la Vida (CBV), nace con la evaluación los resultados de un proyecto dirigido a población joven.

El proyecto fue creado gracias a una iniciativa conjunta entre la agencia patrocinadora y un expresidente del país. Su propósito es construir capital humano y empleabilidad en hombres y mujeres con edades entre 13 y 18 años, desertores o en riesgo de desertar del sistema educativo, sin empleo formal y que viven en ambientes de pobreza y exclusión.

Después de un proceso de licitación pública, se adjudicó la ejecución del proyecto a una de las organizaciones no gubernamentales del país. La implementación se ha realizado en cuatro comunidades seleccionadas por sus condiciones de desventaja social y alto nivel de pobreza, dos pertenecen a zonas urbano marginales, y las otras son del área rural dentro del Valle Central.

Los detalles del proyecto, los nombres de las comunidades, así como los nombres de las instituciones participantes: gobierno, organización ejecutora, agencia patrocinadora, institución encargada de la evaluación de resultados; se mantendrán, como nombres generales, en este artículo ${ }^{2}$.

Como el objetivo principal del programa de intervención consiste en generar empleabilidad en la población meta, se debe tratar de medir este concepto. Los detalles de la operacionalización del concepto Competencias Básicas para la Vida se discuten en el apartado metodológico. El marco teórico de referencia que se utilizó para la medición de empleabilidad y la creación de las Competencias Básicas para la Vida se explica a continuación.

De acuerdo con las definiciones dadas en la literatura se entiende por "empleabilidad" el conjunto de destrezas y atributos generales, asociados a toda clase de trabajos. Estas destrezas son más valoradas por los empleadores que las destrezas específicas o a fines con un puesto, dado que los empleadores creen poder enseñar las destrezas específicas, no así las generales" (AED, 2002).

Sin embargo, la agencia ejecutora, usando la literatura y la experiencia en el trabajo con jóvenes en riesgo social, complementa este concepto de empleabilidad definiéndolo como el "conjunto de competencias que hacen sujeta de inserción en el sector productivo a

\footnotetext{
${ }^{2}$ Para efectos de la creación del indicador, lo importante es el contexto donde surge la necesidad de su construcción y el propósito de la medición. De modo que los detalles del programa de intervención o el diseño de evaluación que se traslapan con la construcción del indicador serán mencionados en este artículo. Otros detalles que puedan ser interés del lector o lectora, deben ser solicitados vía entrevista personal o por correo electrónico a la autora.

${ }^{3}$ Traducción libre de la autora del siguiente texto: "Employability represents general skills and attributes relevant to all kind of jobs. These general skills are valued more by employers than specific job skills, which they believe they can teach on the job" (AED, 2002).
} 
determinada persona, en un tiempo y en un contexto dado, de forma tal que promueva su movilidad social ascendente". (Agencia Ejecutora, 2003).

La Figura 1 especifica los elementos que componen la definición e incluye características del grupo de personas para la cuales la empleabilidad va ser generada, el sector productivo al que pueden incorporarse, el tiempo permitido y el contexto que viven los adolescentes intervenidos.

\section{Figura 1. Particularidades del grupo para el cual la empleabilidad va ser construida, según la Agencia Ejecutora, 2003}

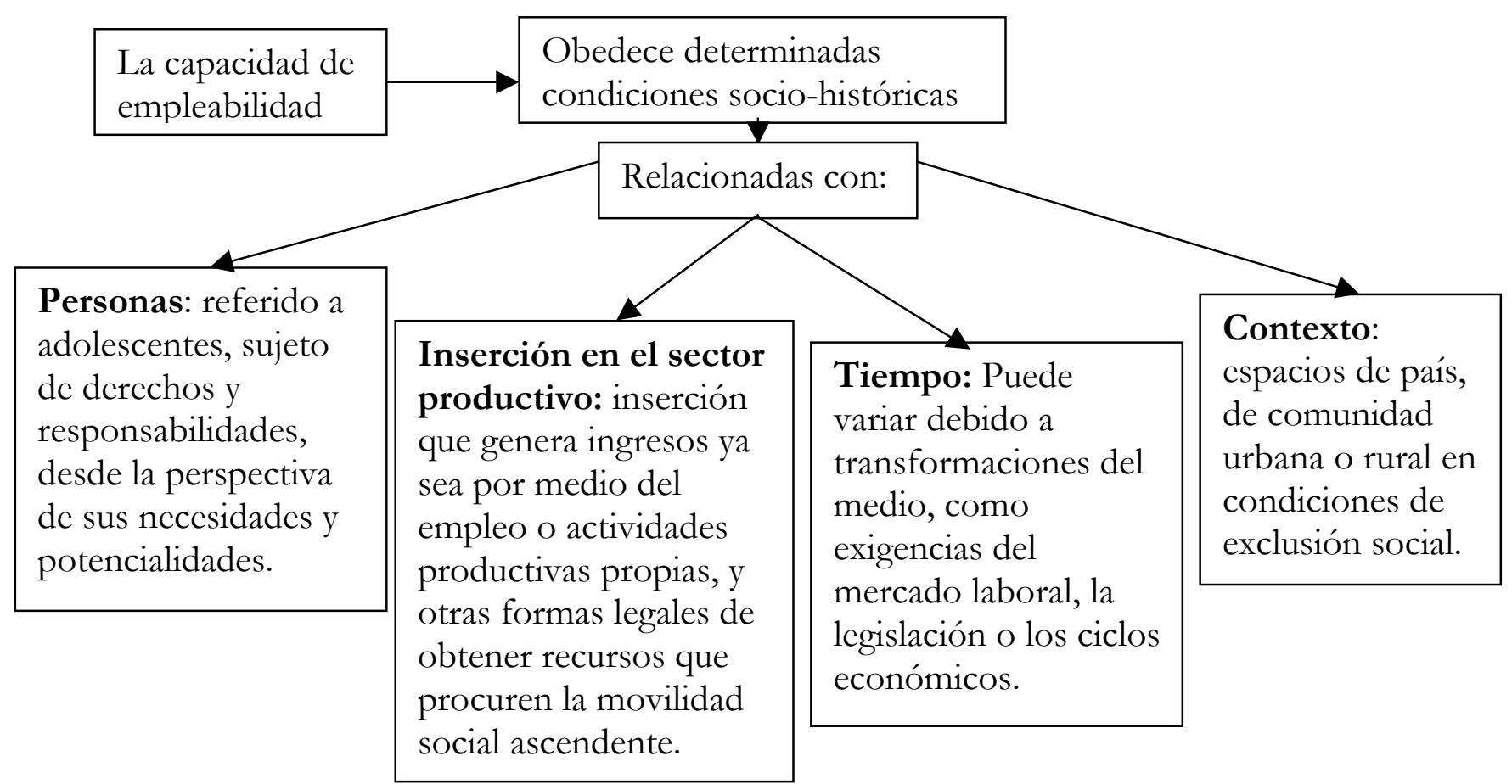

Pero además de las particularidades que la definición adquiere para el grupo de adolescentes, la agencia ejecutora consideró pertinente esclarecer la definición de "competencias de empleabilidad". Estas se definen como un "conjunto de capacidades esenciales para aprender y desempeñarse eficazmente en el puesto de trabajo, que incluye capacidades de comunicación y de la relación interpersonal, de resolución de problemas y manejo de procesos organizacionales" (Agencia Ejecutora, 2003). Las competencias de empleabilidad comprenden conocimientos, habilidades y destrezas como se ilustra en la Figura 2. 


\section{Figura 2. Esquematización de las competencias de empleabilidad, según la Agencia Ejecutora, 2003}

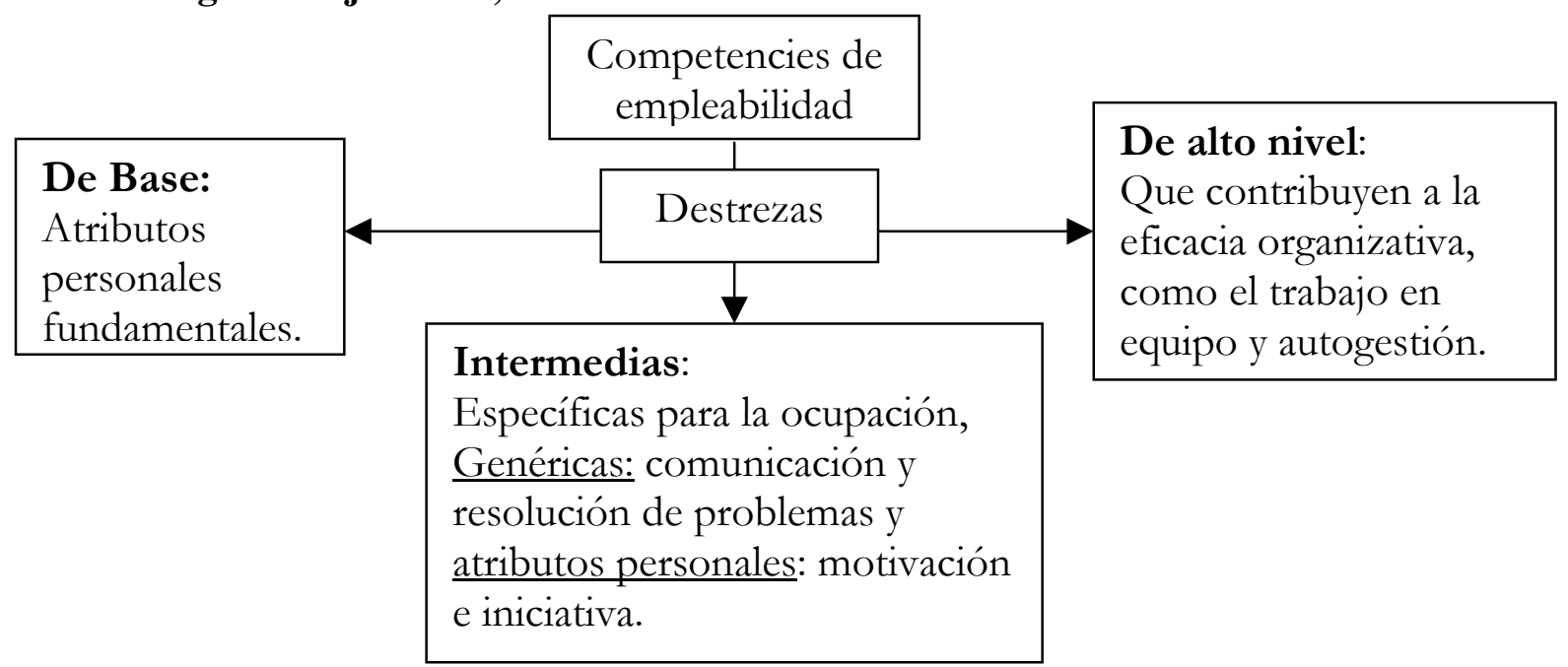

En este punto, es necesario recalcar algunos de los retos de la medición de estos conceptos. Según Babbie (2000), la medición es el resultado "de observaciones cuidadosas y deliberadas del mundo real, cuyo propósito es describir objetos y acontecimientos de acuerdo con los atributos que componen una variable" (Babbie, 2000:97). De este modo, muchos lectores y lectoras, podrían sentirse un poco confundidos ante la definición de empleabilidad y su relación con el indicador de competencias básicas para la vida.

El proceso de medición de estos fenómenos primero tiene que esclarecer los conceptos que las distintas audiencias tienen, así empleabilidad es un término vinculado a las ideas que la agencia ejecutora y la agencia patrocinadora tienen sobre el proyecto.

En principio, la agencia patrocinadora favorecía un concepto de empleabilidad genérico, donde importaban solo las destrezas que los empleadores consideraban importantes. Destrezas como: habilidad para el trabajo en equipo, expresión oral y escrita, capacidad de trabajar bajo presión, capacidad para entender y seguir órdenes,...; siguiendo el esquema de la Figura 2, estas destrezas serían las llamadas intermedias o de alto nivel.

Por otra parte, la agencia ejecutora, defendía la incorporación de las llamadas "destrezas de base", que están vinculadas a atributos personales como: la motivación para hacer tareas y labores; la autoestima; las actitudes hacia los roles de género y el conocimiento de los derechos y deberes laborales, según el código de trabajo y la ley contra el hostigamiento sexual.

Con la incorporación de las destrezas de base, surge así un nuevo concepto "empleabilidad ampliada" que incorpora, la definición tradicional de empleabilidad más la dimensión de los atributos personales fundamentales. 
Una vez obtenido el consenso respecto a lo que se quiere medir, se procede entonces a la conceptualización, que consiste en explicitar las dimensiones del concepto e identificar indicadores ${ }^{4}$.

Después del proceso de conceptuación llevado a cabo entre todas las partes involucradas se establecieron tres dimensiones básicas que componen el indicador de empleabilidad (Figura 3).

La dimensión de competencias básicas para la vida se refiere a aspectos de personalidad asociados con el desempeño del individuo en sus relaciones consigo mismo y con los demás. La dimensión de Educación se refiere al aporte de la educación formal en la construcción de las capacidades que hacen del joven un buen candidato para un empleo. La dimensión de habilidades laborales reúne las destrezas asociadas con el desempeño laboral del joven, y su capacidad de resolución de los problemas cotidianos.

\section{Figura 3. Dimensiones del proceso de conceptualización de empleabilidad}

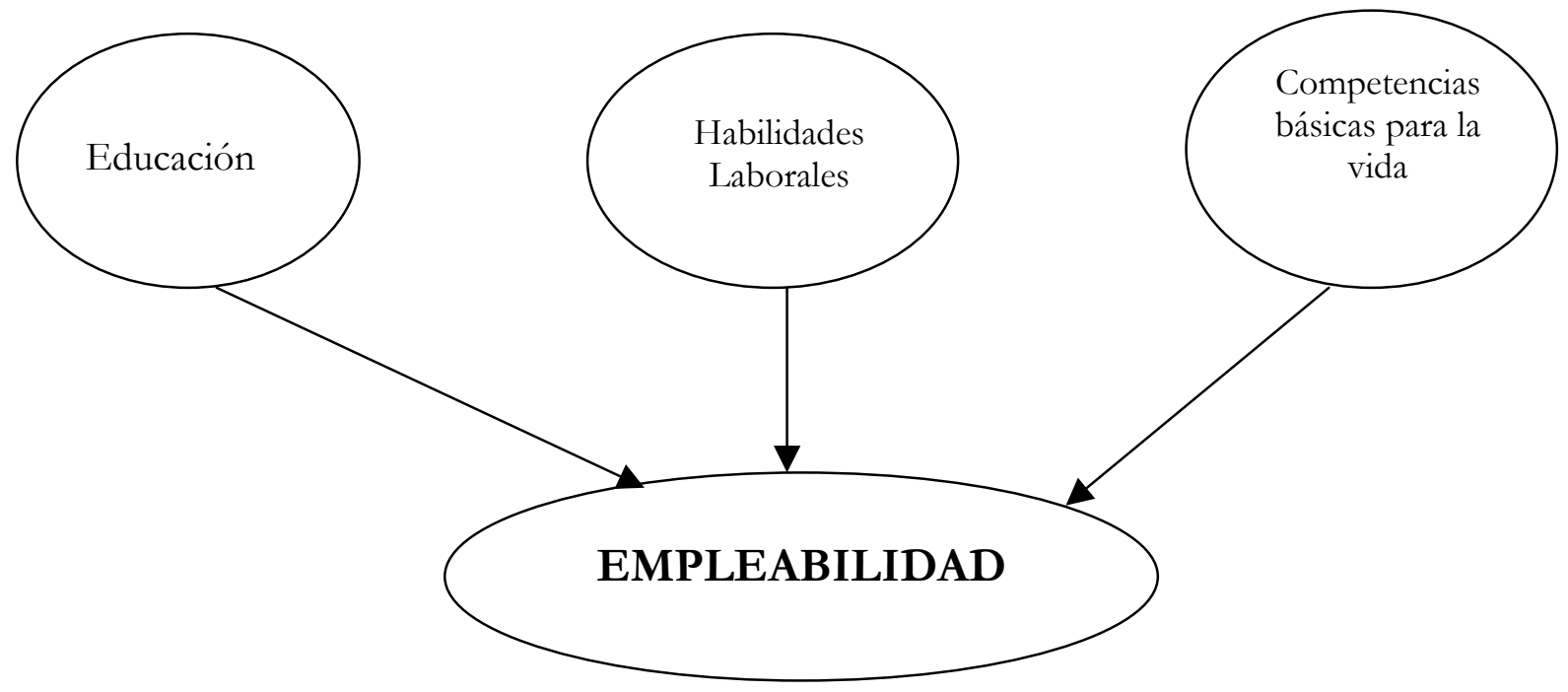

Siguiendo a Babbie (2000), una vez definidas las dimensiones se proponen indicadores para medirlas. Los indicadores representan el modo como una variable o constructo se operacionalizan $^{5}$.

\footnotetext{
${ }^{4}$ Por dimensión se entiende un aspecto o faceta especificable de un concepto, por ejemplo, "empleabilidad ampliada" tiene una dimensión que son las destrezas de base, éstas consideran los atributos personales. Otra dimensión de este concepto son las destrezas técnica, como el uso de un computador.

${ }^{5}$ Una variable es una construcción mental que no requiere de un marco teórico para ser definida, sobre la que hay consenso en general y usualmente es relativamente fácil de medir. Ejemplos: sexo, edad, estatura, peso, talla. En cambio, un constructo es una conceptualización que requiere de marco teórico, por ende es más difícil de cuantificar, veces no hay consenso incluso en cuanto a la definición conceptual. Ejemplos: desarrollo humano, salud, empleabilidad.
} 
Los constructos definidos para operacionalizar la dimensión de competencias básicas para la vida se sustentan en el esquema y objetivos de enseñanza de la agencia ejecutora. En total son seis dimensiones; cinco afectivas llamadas: destrezas interpersonales, relaciones sociales o destrezas intrapersonales, actitud hacia los roles de género, actitud hacia la crítica y actitud hacia la presión en el trabajo, y un constructo de conocimiento sobre derechos laborales y acoso sexual (Figura 4).

Fue de común acuerdo que estos seis constructos sintetizan las competencias básicas que se relacionan con:

i- $\quad$ Competencias de base, como la capacidad de mirarse a sí mismo y poder reconocer sus fortalezas y debilidades, (Destrezas interpersonales); tener conciencia de los roles género (actitudes hacia los roles de género);

ii- $\quad$ Competencias intermedias como la iniciativa, liderazgo, motivación hacia el trabajo (Destrezas interpersonales); más la empatía, la conciencia de ser visto por los demás, la facilidad para establecer relaciones con los demás, entre otras (relaciones sociales);

iii- $\quad$ Competencias de alto nivel, por ejemplo la capacidad de aceptar críticas y corrección, la habilidad para trabajar en equipo y bajo presión; más los conocimientos de los derechos laborales y la ley sobre hostigamiento sexual.

\section{Figura 4. Constructos de la dimensión Competencias Básicas para la Vida}

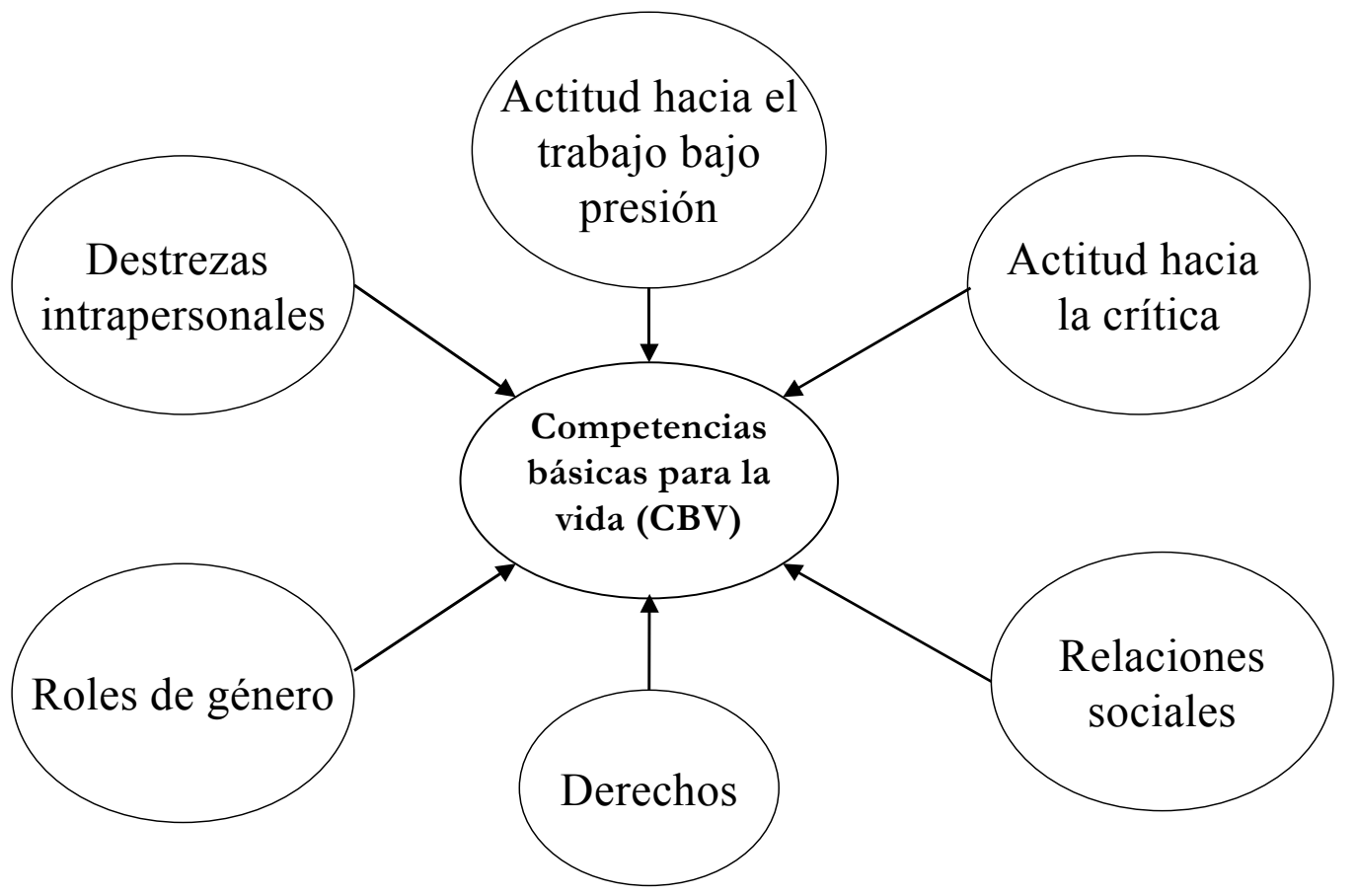


Este artículo se divide en cuatro secciones, incluyendo esta introducción. La siguiente sección esta dedicada a la descripción exhaustiva del procedimiento de operacionalización de los constructos. La sección de resultados muestra las propiedades del indicador y su relación con otras características de los adolescentes. La sección final resume los retos de este tipo de mediciones y se sugiere algunas recomendaciones para el análisis futuro.

\section{METODOLOGÍA}

El estudio del cual deriva este trabajo se encuentra aún en curso, este artículo es el primer esfuerzo por sistematizar la información recolectada en la primera medición hecha durante el primer semestre del año 2004. La metodología que aquí se presenta tiene un carácter más exploratorio que confirmatorio en la validez de constructos. El análisis exploratorio sigue las recomendaciones de Babbie (2000) y Gable y Wolf (1993).

Esta sección está divida en dos partes, la primera se refiere a las características generales de los datos: proceso de recolección, fuente, características de la muestra, etc. La segunda detalla el proceso de análisis para la construcción de los indicadores.

\section{Fuente de datos}

Los datos provienen de una investigación cuyo propósito consiste en evaluar los resultados de una intervención en población joven de comunidades pobres y excluidas. El diseño de la evaluación es longitudinal y cuasiexperimental, tiene dos mediciones en el tiempo y un grupo control que se emparejó según las características sociodemográficas y económicas de las comunidades, reportadas en el último Censo de Población y Vivienda (2000).

Los datos que se utilizan en este artículo provienen de la primera etapa de recolección, hecha entre los meses de enero a junio del año 2004. La técnica de recolección fue una entrevista personal realizada con un cuestionario precodificado. Los criterios de selección para los informantes fueron: hombre o mujer con edades entre los 13 y 18 años cumplidos, con sexto grado de educación primaria cursado. Estos criterios fueron establecidos y fundamentados por la agencia ejecutora.

La composición de la muestra según sexo, edad, condición de estudio, condición de trabajo, tipo de comunidad (si es principalmente urbana o rural) se presenta en el Cuadro 1. 


\section{Cuadro 1. Composición de la muestra de adolescentes}

\begin{tabular}{lc}
\hline \multicolumn{1}{c}{ Variables } & \% \\
\hline \hline Entrevistados (n) & 977 \\
Sexo & \\
Masculino & \\
Femenino & 44,3 \\
Edad & 55,7 \\
Menor de 15 & \\
15 y más & 33,7 \\
Zona & 66,3 \\
Rural & \\
Urbana & \\
Estaba estudiando * & 42,1 \\
& 57,9 \\
\hline
\end{tabular}

*Durante el ciclo lectivo del 2003

\section{Operacionalización}

El conjunto de ítems (enunciados o variables), que miden cada uno de los constructos que componen la dimensión de CBV siguió un proceso delicado de elaboración. La mayoría fueron instrumentos creados con otros propósitos y que fueron adecuados a las necesidades de la evaluación y a los objetivos de medición del proyecto.

Los constructos afectivos fueron precodificados usando una escala de Likert de cinco categorías. Los y las entrevistadas debían responder si estaban: totalmente en desacuerdo, en desacuerdo, ni en desacuerdo ni en acuerdo o totalmente de acuerdo. El constructo de conocimiento tenía tres tipos de respuesta "Verdadero", "Falso", "No sabe", de estas opciones solo una es la respuesta correcta.

Cada constructo se representó en una escala independiente; su ubicación dentro del cuestionario, así como la cantidad de ítems necesarios para representarlo fue sometida a distintas pruebas. Todos los constructos pasaron por lo menos por tres fases de validación:

i-validación facial: adecuación del lenguaje y los ejemplos según las características de la población entrevistada;

ii- validación de expertos, expertos en psicometría y trabajo con adolescentes más los representantes de la agencia ejecutora valoraron cada uno de los ítems y los juzgaron bajo seis criterios: 1) redacción (precisa, específica), 2) adecuación del 
vocabulario y contexto a la población de interés, 3) se refiere solo a una cosa (unidimensional), 4) poder discriminatorio (diferencia entre las posiciones de las distintas personas), 5) direccionalidad en términos de lo que se quiere medir; 6) familiaridad, refleja situaciones cotidianas de los adolescentes;

iii- validación empírica, prueba piloto del instrumento en una población con características similares y con un tamaño de muestra no menor a 100 adolescentes.

Una vez colectados y procesados los datos, la validación del indicador siguió los siguientes pasos:

1- Establecer el comportamiento de los valores faltantes.

2- $\quad$ Recodificación de las variables (ítems) para que indiquen la misma dirección

3- Imputación de los valores faltantes de las variables (ítems) e individuos

4- $\quad$ Estandarización de las variables (ítems)

5- $\quad$ Análisis de factores

6- $\quad$ Análisis de confiabilidad

Para establecer el comportamiento de los valores faltantes, primero se analizaron las distribuciones de frecuencias simples de cada una de las variables (ítems) que pertenecen a un constructo. El criterio de decisión fue excluir del análisis toda variable que tuviera menos del $80 \%$ de las respuestas. Además se decidió excluir a todo individuo (observación) que hubiese contestado menos del 40\% de los ítems dentro de una escala.

La recodificación de las variables, se hizo con el propósito que los valores vayan en la misma dirección. Cuando se mide conocimientos la recodificación se hace para asignarle el mismo valor todas las respuestas correctas.

Representar la misma dirección significa, que si el muchacho o la muchacha contestó por ejemplo, "Totalmente en desacuerdo" al ítem: "Los hombres que hacen aeróbicos son playos" y este ítem está midiendo actitudes hacia los roles de genero, el valor de la respuesta debe reflejar el máximo puntaje (5), pues a mayor puntaje mejor es la actitud hacia los roles de género.

En el caso de conocimientos, si el joven respondió "Verdadero" a un enunciado como "Si un adolescente no está asegurado por su cuenta o por cuenta de sus papás, el Gobierno debe brindarle el seguro" debe asignársele el valor de 1 (uno) a su respuesta correcta y 0 (cero) en cualquier otro caso.

La imputación de los valores faltantes por individuos se hizo según el valor promedio que el mismo individuo obtuvo tomando en cuenta los ítems que respondió en cada escala (no menos del 60\%). Al imputar los valores faltantes en cada individuo se remedia el problema de los valores faltantes por variable. 
Una vez que se ha hecho la imputación de los valores faltantes y antes de realizar cualquier análisis, las variables deben estandarizarse, porque se quiere que todas tengan la misma importancia en los análisis psicométricos posteriores.

La estandarización es "estrictamente necesaria" cuando en un mismo constructo los ítems tienen distintas escalas, por ejemplo, unos ítems tiene una escala de Likert de tres categorías, mientras otros tiene una escala de cinco o una escala de siete (Jonhson, 1998). En el caso de los constructos que forman el indicador de CBV este paso puede ser omitido porque todas las escalas afectivas tienen cinco categorías. Solo la escala de conocimientos tiene tres, pero esta se analiza por aparte.

Con las variables estandarizadas se hace el análisis de factores en dos pasos. Primero se usa el porcentaje de varianza explicada para definir los factores. El criterio dice que cuando este porcentaje es mayor o igual al 10\% se define un factor (Anastasi \& Urbina, 1998). Por ejemplo, si el porcentaje de variancia presenta la siguiente distribución: primero $35 \%$, luego $15 \%$, luego $9 \%$ y luego $8.5 \%$; se dice que el constructo tiene dos factores o dimensiones importantes.

El criterio del porcentaje de varianza explicada se complementa con un gráfico llamado "Diagrama de Sedimentación". La interpretación de este gráfico dice que un constructo tiene tantas dimensiones como eje valores existan antes del punto de máxima inflexión (codo) (Anastasi \& Urbina, 1998).

Si los constructos son unidimensionales, luego del análisis de factores se pasa al análisis de confiabilidad. Si los constructos presentan más de una dimensión, se hace un segundo análisis de factores estableciendo el número específico de factores usando las cargas factoriales de una matriz rotada oblicua. La rotación usa para clasificar cada uno de los ítems en la dimensión o factor con más carga factorial y así buscar el sentido teórico de la solución resultante de acuerdo con el marco teórico (Jonhson, 1998).

El análisis de confiabilidad se hace con el coeficiente de correlación "Alfa de Cronbach". Hasta donde sea posible y sin perjudicar o modificar el constructo, se trata de maximizar el valor de Alfa eliminando ítems que no contribuyan a la confiabilidad. Los ítemes son eliminados cuando su índice de discriminación o la correlación entre el ítem y el total de ítems que componen el constructo es menor a 0,30. La eliminación de los ítems con baja calidad técnica se hace uno a uno para estudiar con cuidado el proceso de optimización del “Alfa" (Nunnally \& Bernstein, 1995).

El "Alfa" alcanza un valor aceptable cuando es superior a 0,7. Si los ítems que miden un constructo no alcanzan este valor, el indicador no se puede tratar como una escala. Cuando el conjunto de ítems que miden el constructo obtiene un alfa superior a $0,7 \mathrm{se}$ puede sumar el valor de cada ítem o calcular el promedio, para así construir un indicador sintético que represente el constructo (Nunnally \& Bernstein, 1995). 
Este procedimiento, se realizó para cada conjunto de ítems que representan cada uno de los constructos de la dimensión $\mathrm{CBV}^{6}$.

\section{RESULTADOS GENERALES}

Los resultados serán presentados según los seis pasos descritos en la sección anterior (Operacionalización). Para ello se agruparon estos pasos en tres subtítulos: i- Valores faltantes y transformación de variables, ii- Análisis de la dimensionalidad de los datos, iii- Análisis de Confiabilidad. El cuarto subtítulo muestra algunas propiedades del indicador.

\section{Valores faltantes y transformación de variables}

Ninguno de los ítems tenía más del $20 \%$ de valores faltantes. El número máximo de ítems pertenecientes al mismo constructo, que quedaron sin contestar fue cinco. No obstante, son pocos los ítems con 100\% de respuesta. El Cuadro 2 muestra el número de ítems que componen cada constructo, el número de ítems con valores faltantes y su rango; en la última columna, el número máximo de ítems sin responder.

\section{Cuadro 2. Reporte de los valores faltantes por individuo según conjuntos de ítems} de un mismo constructo

\begin{tabular}{lcccc}
\hline & $\begin{array}{c}\text { Número de } \\
\text { ítems }\end{array}$ & $\begin{array}{c}\text { Número de } \\
\text { ítems con } \\
\text { valores } \\
\text { faltantes }\end{array}$ & $\begin{array}{c}\text { Rango de } \\
\text { valores } \\
\text { faltantes por } \\
\text { individuo }\end{array}$ & $\begin{array}{c}\text { Número de } \\
\text { ítems con el } \\
\text { faltante } \\
\text { máximo }\end{array}$ \\
\hline Destrezas intrapersonales & 18 & 13 & $1-2$ & 4 \\
Actitud hacia la crítica & 7 & 4 & $1-3$ & 1 \\
Actitud hace el trabajo bajo presión & 15 & 6 & $1-2$ & 4 \\
Relaciones Sociales & 16 & 10 & $1-3$ & 3 \\
Actitud hacia los roles de género & 9 & 4 & $1-5$ & 1 \\
\hline
\end{tabular}

El Cuadro 2 refleja un bajo porcentaje de no respuesta. Al hacer el análisis por variable, por ejemplo, 18 ítems representan el constructo "Destrezas interpersonales", de esos 72\% tiene al menos un valor faltante, pero la cantidad de valores faltante no es mayor que tres, lo que equivale a un porcentaje de no respuesta menor a $0,20 \%$. Además el número máximo de ítems con ese porcentaje es cuatro, lo que indica que los nueve restantes tienen $0,10 \%$ de no respuesta.

\footnotetext{
${ }^{6}$ Los datos se procesaron en el paquete estadístico Stata Versión 8 SE. Para el análisis se generó un programa cuyos comandos e instrucciones generales pueden ser solicitadas a la autora.
} 
Al hacer el análisis por individuos no se encontró un solo caso que tuviera un porcentaje de no respuesta mayor a $10 \%$ en los ítems de una misma escala. Con esta evidencia se hizo a la imputación, luego a la recodificación y estandarización de las variables.

\section{Análisis de la dimensionalidad de los datos}

Para probar la dimensionalidad de cada constructo se realizó un análisis de factores por componentes comunes, con una extracción factorial cuando el eje valor era mayor que la unidad. Se aplicó una solución de componentes comunes y no componente principales porque inicialmente no se supone independencia entre los factores. Además no se puede afirmar que la variabilidad de cada uno de los ítems está completamente explicada por la variabilidad del constructo, por lo que se utiliza el cuadrado de la correlación múltiple entre el constructo y el ítem para estimar la comunalidad (no se puede asumir que ésta es igual a la unidad).

El análisis de factores complementado con el diagrama de sedimentación verificaron la unidimensionalidad de los factores excepto en el constructo que mide "Derechos laborales y hostigamiento sexual". Por distintas razones se decidió no incluir este indicador dentro del indicador sintético del CBV.

El Cuadro 3 y la Figura 5 muestran el uso e interpretación del análisis de factores y el diagrama de sedimentación para el caso del indicador de destrezas intrapersonales.

Usando el criterio de la proporción de varianza explicada aparecen, en el Cuadro 3, tres factores con más del 10\% (cuarta columna, números en negrita). No obstante, el primer factor explica una altísima proporción de la variabilidad (82.20\%) y esta evidencia se complementa con el valor de los eje valores que aparecen en la segunda columna, solo uno es superior a las unidad.

Al contrastar la evidencia del Cuadro 3 con el Diagrama de Sedimentación (Figura 5) se remarca la diferencia entre el primer factor con los anteriores. Se puede decir que el punto máximo de inflexión se ubica en el segundo factor, por eso se concluye unidimensionalidad.

Este mismo procedimiento se siguió para verificar la validez de constructo de las otras cuatro subdimensiones actitudinales: actitud hacia la crítica, actitud hacia el trabajo bajo presión, relaciones sociales (destrezas interpersonales) y actitud hacia los roles de género. 
Cuadro 3. Resultados del un análisis de factores por componentes comunes para el conjunto de ítems del constructo destrezas intrapersonales

\begin{tabular}{rrrrr}
\hline \multicolumn{1}{r}{ Factor } & Eigenvalue & Difference & Proporción & Cumulative \\
\hline \hline 1 & $\mathbf{2 . 5 4 9 3}$ & 1.8672 & $\mathbf{0 . 8 2 2 0}$ & 0.8220 \\
2 & 0.6822 & 0.0674 & $\mathbf{0 . 2 2 0 0}$ & 1.0419 \\
3 & 0.6147 & 0.3188 & $\mathbf{0 . 1 9 8 2}$ & 1.2402 \\
4 & 0.2959 & 0.0638 & 0.0954 & 1.3356 \\
5 & 0.2320 & 0.1570 & 0.0748 & 1.4104 \\
6 & 0.0750 & 0.0277 & 0.0242 & 1.4346 \\
7 & 0.0473 & 0.0308 & 0.0153 & 1.4498 \\
8 & 0.0166 & 0.0230 & 0.0053 & 1.4552 \\
9 & -0.0065 & 0.0451 & -0.0021 & 1.4531 \\
10 & -0.0515 & 0.0204 & -0.0166 & 1.4365 \\
11 & -0.0719 & 0.0331 & -0.0232 & 1.4133 \\
12 & -0.1051 & 0.0408 & -0.0339 & 1.3794 \\
13 & -0.1458 & 0.0180 & -0.0470 & 1.3324 \\
14 & -0.1638 & 0.0036 & -0.0528 & 1.2796 \\
15 & -0.1674 & 0.0376 & -0.0540 & 1.2256 \\
16 & -0.2050 & 0.0267 & -0.0661 & 1.1595 \\
17 & -0.2317 & 0.0313 & -0.0747 & 1.0848 \\
18 & -0.2630 & & -0.0848 & 1.0000 \\
\hline
\end{tabular}

Figura 5. Diagrama de Sedimentación para el conjunto de ítems del constructo destrezas intrapersonales




Como el análisis es de carácter exploratorio, se realizaron tres extracciones rotadas para dos y tres factores, al verificar las cargas factoriales no se confirmó el sentido teórico de ninguna de las subdimensiones. Si en cambio el marco teórico confirma la existencia de una única dimensión, lo que da mayor confianza a la solución unifactorial.

\section{Análisis de Confiabilidad}

Comprobada la unidimensionalidad de cada uno de las subdimensiones del indicador de CBV, se procedió al análisis de confiabilidad usando el proceso de maximización del coeficiente de correlación "Alfa de Cronbach".

En la operacionalización se dijo que este proceso consiste en calcular la correlación entre el conjunto de ítems y el constructo. Luego se verifica la calidad técnica de cada uno de estos ítems con la correlación total que tendría el indicador si el ítem es borrado.

Los Cuadro 4, Cuadro 5 y Cuadro 6 ilustran el proceso de optimización del alfa para el conjunto de ítemes que operacionalizan el constructo "Destrezas interpersonales".

\section{Cuadro 4. Solución inicial del Alfa de Cronbach y otros estadísticos asociados para el conjunto de ítems del constructo destrezas intrapersonales}

\begin{tabular}{lcccccc}
\hline Ítem & Obs. & Sing. & $\begin{array}{c}\text { average } \\
\text { ítem-test } \\
\text { correlación }\end{array}$ & $\begin{array}{c}\text { ítem-rest } \\
\text { correlación }\end{array}$ & $\begin{array}{c}\text { Inter.- } \\
\text { ítem } \\
\text { covariance }\end{array}$ & alpha \\
\hline \hline & & & & & & \\
intra_01 & 977 & + & 0.2820 & 0.1529 & 0.1317 & 0.7206 \\
intra_02 & 977 & + & 0.2969 & 0.1686 & 0.1309 & 0.7191 \\
intra_03 & 977 & + & 0.3244 & 0.1978 & 0.1294 & 0.7164 \\
intra_04 & 977 & + & 0.4136 & 0.2939 & 0.1245 & 0.7073 \\
intra_05 & 977 & + & 0.3101 & 0.1825 & 0.1302 & 0.7178 \\
intra_06 & $\mathbf{9 7 7}$ & + & $\mathbf{0 . 2 6 8 6}$ & $\mathbf{0 . 1 3 8 8}$ & $\mathbf{0 . 1 3 2 4}$ & $\mathbf{0 . 7 2 1 9}$ \\
intra_07 & 977 & + & 0.4708 & 0.3571 & 0.1213 & 0.7012 \\
intra_08 & 977 & + & 0.3646 & 0.2408 & 0.1272 & 0.7124 \\
intra_09 & 977 & + & 0.4547 & 0.3392 & 0.1222 & 0.7030 \\
intra_10 & 977 & + & 0.4517 & 0.3359 & 0.1224 & 0.7033 \\
intra_11 & 977 & + & 0.5125 & 0.4038 & 0.1190 & 0.6967 \\
intra_12 & 977 & + & 0.4084 & 0.2883 & 0.1248 & 0.7079 \\
intra_13 & 977 & + & 0.4909 & 0.3795 & 0.1202 & 0.6990 \\
intra_14 & 977 & + & 0.5106 & 0.4016 & 0.1191 & 0.6969 \\
intra_15 & 977 & + & 0.5183 & 0.4103 & 0.1187 & 0.6960 \\
intra_16 & 977 & + & 0.4616 & 0.3469 & 0.1218 & 0.7022 \\
intra_17 & 977 & + & 0.5030 & 0.3930 & 0.1195 & 0.6977 \\
intra_18 & 977 & + & 0.4436 & 0.3269 & 0.1228 & 0.7042 \\
Test scale & & & & & & \\
\hline
\end{tabular}


El Cuadro 4 resalta en negrita el valor inicial del estadístico alfa de Cronbach cuando la escala se compone de 18 ítems. El proceso de optimización consiste en encontrar aquel ítem cuya correlación entre el ítem y el constructo sea menor que 0,3 pero que a su vez represente el máximo valor del Alfa, si el ítem es borrado. El primer ítem que no reúne la calidad técnica requerida es el número 6 (marcado en negrita). Si ese ítem se elimina de la escala la correlación de los demás ítems y el constructo alcanzaría un valor de $\sqrt{0,72}$. El Cuadro 5 presenta los resultados cuando el ítem 6 es excluido de la escala.

\section{Cuadro 5. Ilustración del proceso de maximización del “Alfa de Cronbach” al eliminar el ítem con peor calidad técnica}

\begin{tabular}{|c|c|c|c|c|c|c|}
\hline Ítem & Obs. & Sing. & $\begin{array}{c}\text { ítem-test } \\
\text { correlación }\end{array}$ & $\begin{array}{c}\text { ítem-rest } \\
\text { correlación }\end{array}$ & $\begin{array}{c}\text { average } \\
\text { Inter.- } \\
\text { ítem } \\
\text { covariance } \\
\end{array}$ & alpha \\
\hline intra_01 & 977 & + & 0.2552 & 0.1210 & 0.1429 & 0.7274 \\
\hline intra_02 & 977 & + & 0.2768 & 0.1437 & 0.1416 & 0.7253 \\
\hline intra_03 & 977 & + & 0.3258 & 0.1955 & 0.1387 & 0.7204 \\
\hline intra_04 & 977 & + & 0.4159 & 0.2928 & 0.1332 & 0.7109 \\
\hline intra_05 & 977 & + & 0.3167 & 0.1858 & 0.1392 & 0.7213 \\
\hline intra_07 & 977 & + & 0.4753 & 0.3586 & 0.1296 & 0.7043 \\
\hline intra_08 & 977 & + & 0.3708 & 0.2438 & 0.1359 & 0.7157 \\
\hline intra_09 & 977 & + & 0.4616 & 0.3433 & 0.1304 & 0.7059 \\
\hline intra_10 & 977 & + & 0.4638 & 0.3458 & 0.1303 & 0.7056 \\
\hline intra_11 & 977 & + & 0.5244 & 0.4139 & 0.1266 & 0.6987 \\
\hline intra_12 & 977 & + & 0.4097 & 0.2861 & 0.1336 & 0.7115 \\
\hline intra_13 & 977 & + & 0.5037 & 0.3904 & 0.1279 & 0.7011 \\
\hline intra_14 & 977 & + & 0.5095 & 0.3969 & 0.1275 & 0.7005 \\
\hline intra_15 & 977 & + & 0.5326 & 0.4232 & 0.1261 & 0.6978 \\
\hline intra_16 & 977 & + & 0.4714 & 0.3542 & 0.1298 & 0.7048 \\
\hline intra_17 & 977 & + & 0.5158 & 0.4041 & 0.1271 & 0.6997 \\
\hline intra_18 & 977 & + & 0.4529 & 0.3337 & 0.1310 & 0.7068 \\
\hline Test scale & & & & & 0.132445 & 0.7219 \\
\hline
\end{tabular}

Al ser eliminado el ítem con la peor calidad técnica, los coeficientes de correlación se recalculan. De este modo se vuelve a buscar el ítem con la correlación más baja, que al ser eliminado produciría el alfa más alto; ese ítem es el número 3 (en negrita en el Cuadro 5). 
El proceso de eliminación continua hasta alcanzar el mayor valor posible del alfa sin afectar el constructo medido. Nno es recomendable medir constructos tan complejos con menos de cinco ítems.

En ocasiones el máximo alfa posible se estabiliza, es decir, cualquier otro ítem que sea eliminado de la escala disminuirá el valor del alfa. Este fue el caso de todos los constructos medidos en el artículo, en el ejemplo de las Destrezas intrapersonales, el alfa se estabiliza con 13 ítems.

El Cuadro 6 en su última columna muestra que cualquier ítem que sea eliminado de la escala disminuiría el valor de alfa. El ejemplo más claro lo representa el ítem 15, si ese ítem es eliminado de la escala, el alfa resultante sería aun menor que el alfa inicial.

Cuadro 6. Solución final del Alfa de Cronbach y otros estadísticos asociados para el conjunto de ítems del constructo destrezas intrapersonales

\begin{tabular}{|c|c|c|c|c|c|c|}
\hline Ítem & Obs. & Sing. & $\begin{array}{c}\text { ítem-test } \\
\text { correlación }\end{array}$ & $\begin{array}{c}\text { ítem-rest } \\
\text { correlación }\end{array}$ & $\begin{array}{c}\text { average } \\
\text { Inter.- } \\
\text { ítem } \\
\text { covariance } \\
\end{array}$ & alpha \\
\hline intra_04 & 977 & + & 0.4225 & 0.2817 & 0.1853 & 0.7319 \\
\hline intra_07 & 977 & + & 0.4901 & 0.3575 & 0.1788 & 0.7232 \\
\hline intra_08 & 977 & + & 0.3662 & 0.2199 & 0.1908 & 0.7388 \\
\hline intra_09 & 977 & + & 0.4812 & 0.3474 & 0.1796 & 0.7243 \\
\hline intra_10 & 977 & + & 0.4817 & 0.3480 & 0.1796 & 0.7243 \\
\hline intra_11 & 977 & + & 0.5146 & 0.3854 & 0.1764 & 0.7199 \\
\hline intra_12 & 977 & + & 0.4222 & 0.2813 & 0.1854 & 0.7319 \\
\hline intra_13 & 977 & + & 0.5548 & 0.4319 & 0.1725 & 0.7144 \\
\hline intra_14 & 977 & + & 0.5405 & 0.4153 & 0.1739 & 0.7164 \\
\hline intra_15 & 977 & + & 0.5675 & 0.4466 & 0.1713 & 0.7127 \\
\hline intra_16 & 977 & + & 0.5221 & 0.3940 & 0.1757 & 0.7189 \\
\hline intra_17 & 977 & + & 0.5423 & 0.4174 & 0.1737 & 0.7161 \\
\hline intra_18 & 977 & + & 0.4870 & 0.3539 & 0.1791 & 0.7236 \\
\hline Test scale & & & & & 0.1786195 & 0.7387 \\
\hline
\end{tabular}

El análisis de confiabilidad para los otros indicadores no necesitó de procesos de maximización del "Alfa de Cronbach", puesto que con el conjunto de ítems propuesto el coeficiente se estabilizaba. 
El Cuadro 7 muestra los coeficientes "alfa" iniciales y finales para cada componente. En la segunda columna, se comparan los resultados del alfa final obtenido con los datos de la prueba piloto, las otras columnas se refiere a los datos de la primera medición.

\section{Cuadro 7. Comparación del Alfa de Cronbach para los distintos componentes del indicador CBV}

\begin{tabular}{|c|c|c|c|c|c|}
\hline Dimensión & Piloto & $\begin{array}{l}\text { Alfa } \\
\text { Inicial }\end{array}$ & $\begin{array}{c}\text { Número } \\
\text { inicial de } \\
\text { ítems }\end{array}$ & $\begin{array}{l}\text { Alfa } \\
\text { Final }\end{array}$ & $\begin{array}{c}\text { Número } \\
\text { final de } \\
\text { ítems }\end{array}$ \\
\hline Destrezas intrapersonales & 0.6727 & 0.7188 & 18 & 0.7387 & 13 \\
\hline Actitud hacia la crítica & 0.6711 & 0.2740 & 7 & & \\
\hline $\begin{array}{l}\text { Actitud hace el trabajo bajo } \\
\text { presión }\end{array}$ & 0.6822 & 0.7971 & 15 & 0.7971 & 15 \\
\hline Relaciones Sociales & 0.6601 & 0.7568 & 16 & 0.7568 & 16 \\
\hline Actitud hacia los roles de género & 0.8171 & 0.8578 & 9 & 0.8578 & 9 \\
\hline
\end{tabular}

\section{Cálculo y análisis del indicador obtenido}

Con los resultados obtenidos en el análisis de confiabilidad se generan indicadores sintéticos que corresponde al valor promedio de cada uno de los ítemes estandarizados para cada constructo. Se construyeron indicadores para los constructos de destrezas intrapersonales (destrezas), trabajo de presión (presión), relaciones sociales (sociales) y actitudes hacia los roles de género (roles).

La escala de actitud ante la crítica no se construyó, dado que su confiabilidad es muy baja. El valor del alfa obtenido $(0,2740)$ muestra que la escala no es confiable; incluirla dentro del indicador CBV implicaría agregar error de medición.

Las correlaciones entre las escalas que pertenecen al índice de CBV se muestran en el Cuadro 8. La correlación más alta, es de 0,46 y representa la asociación lineal positiva entre las destrezas intrapersonales y las relaciones sociales o destrezas interpersonales. Este resultado es consistente con evidencias empíricas anteriores. No obstante, como las correlaciones no son mayores a 0,5 se puede asumir que los indicadores son independientes, lo cual permitiría generar una escala aditiva para sintetizar estas subdimensiones en el índice de CBV. 
Cuadro 8. Correlación entre los indicadores

\begin{tabular}{lrrrr}
\hline & destrezas & presión & sociales & roles \\
destrezas & 1 & & & \\
presión & 0.214 & 1 & & \\
sociales & 0.426 & 0.100 & 1 & \\
roles & 0.137 & 0.200 & 0.203 & 1 \\
\hline
\end{tabular}

El puntaje obtenido mediante la suma de los indicadores mostró un comportamiento simétrico con una distribución similar a la normal. (Figura 6). Los valores extremos se encuentran en valores bajos y la moda se concentra alrededor de la primera desviación estándar.

\section{Figura 6. Histograma del indicador de CBV}

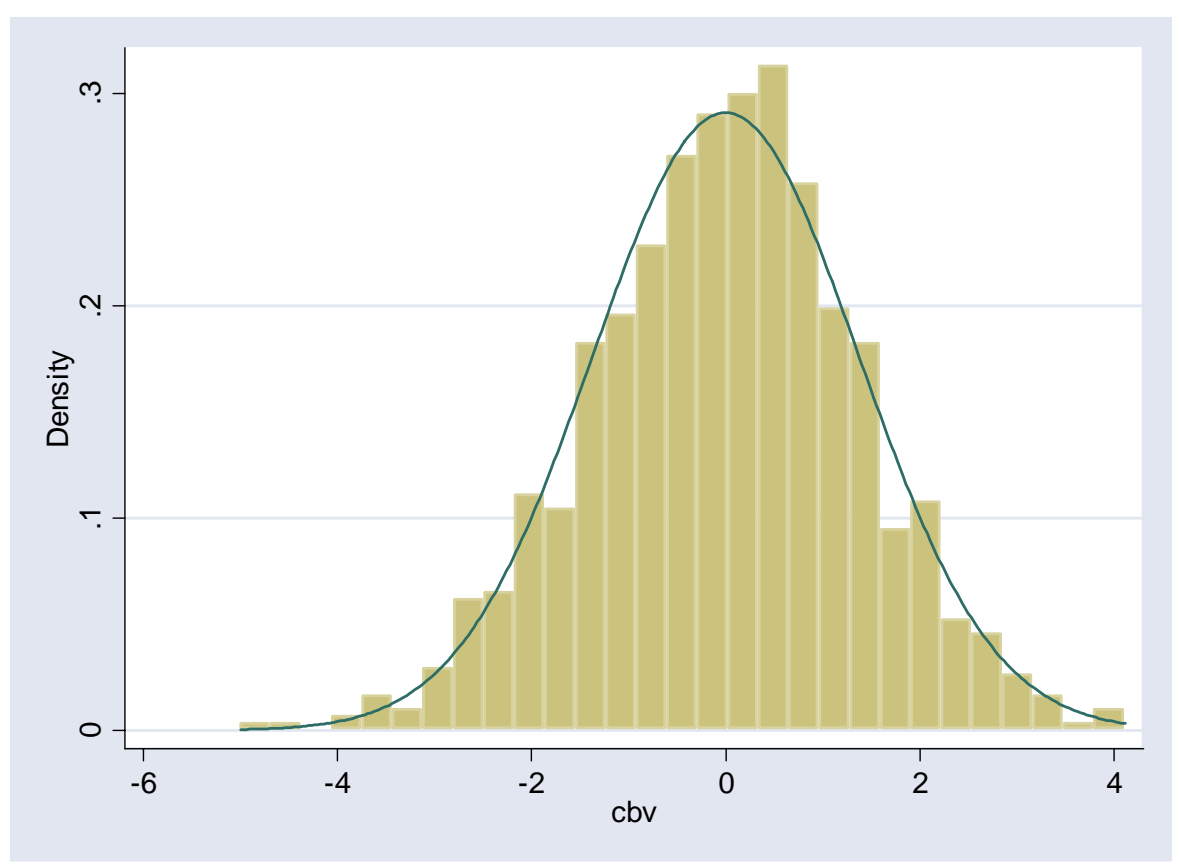

Este comportamiento relativamente simétrico resulta ventajoso porque permite distinguir mejor a los sujetos que se encuentran por encima y por debajo del promedio y de algún modo favorece la aplicación de las pruebas de comparación de medias más comunes.

Para efectos ilustrativos, se categorizó el indicador en tres niveles, usando un criterio netamente estadístico. El puntaje promedio para cada una de estas categorías es 1.507365 para niveles bajos, 0.0434862 para niveles intermedios y 1.468383 en niveles altos. 
Al comparar los valores medios entre las principales subclases de la muestra no se encontraron diferencias estadísticamente significativas por sexo, ni por zona, si en cambio por grupos de edad y por la asistencia a la educación formal. Los jóvenes de 15 años y más tienen un puntaje promedio más alto que los menores de 15 años, lo mismo sucede con los que estudian (Cuadro 9).

\section{Cuadro 9. Pruebas de diferencias de promedios para distintas características de la muestra suponiendo igual de varianza}

\begin{tabular}{lcc}
\hline \multicolumn{1}{c}{ Variables } & $\begin{array}{c}\text { Promedio del } \\
\text { CBV }\end{array}$ & $\begin{array}{c}\text { Hipótesis y } \\
\text { Probabilidad }\end{array}$ \\
\hline \hline & & \\
Sexo & & Ha: dif $\neq 0$ \\
Masculino & 0,009 & $\mathrm{t}=0,2406$ \\
Femenino & 0,012 & $\mathrm{P}>|\mathrm{t}|=0,809$ \\
Edad & & $\mathrm{Ha}: \operatorname{dif} \neq 0$ \\
Menor de 15 & -0.302 & $\mathrm{t}=-4,961$ \\
15 y más & 0.153 & $\mathbf{P}>|\mathbf{t}|=\mathbf{0 , 0 0}$ \\
& & \\
Zona & & $\mathrm{Ha}: \operatorname{dif} \neq 0$ \\
Rural & 0,038 & $\mathrm{t}=0,744$ \\
Urbana & $-0,023$ & $\mathrm{P}>|\mathrm{t}|=0,477$ \\
& & \\
Estaba estudiando $*$ & & $\mathrm{Ha}:$ dif $>0$ \\
Si & $-0,527$ & $\mathrm{t}=-5,208$ \\
No & 0,096 & $\mathbf{P}>|\mathbf{t}|=\mathbf{1 , 0 0 0}$ \\
& & \\
\hline
\end{tabular}

*Durante el ciclo lectivo del 2003

\section{DISCUSIÓN}

Los resultados presentados permiten construir y replicar un indicador que mide competencias básicas para la vida, un concepto que refleja destrezas de empleabilidad de base, intermedias y avanzadas y que se asocia con atributos personales fundamentales y a actitudes del adolescente dentro del entorno social al que pertenece.

La construcción del indicador uso de técnicas psicométricas para la validación de las distintas subdimensiones. Según el marco conceptual este indicador está compuesto por seis dimensiones, la validación empírica hecha en este artículo incluye solamente cuatro de ellas, todas de carácter actitudinal. Se eliminó la subdimensión de actitudes hacia la crítica por tener una baja confiabilidad y no se incluyó la escala de conocimientos sobre Derechos Laborales. 
El indicador posee algunas ventajas en términos de la capacidad de discriminación debido a que su distribución tiende a ser normal. Sin embargo se ve afectaba por valores extremos a la izquierda del promedio y valores modales a una desviación estándar (positiva) del promedio. Esta distribución servirá para discusiones futuras sobre los puntos de corte (baremos) y el número de categorías que debería tener, o si para efectos de la interpretación se prefiera trabajar con niveles (alto, medio, bajo) o se prefiera la medición (variable) continua.

Falta analizar cada una de las subdimensiones y su correlación con la escala de "Deseabilidad Social". La deseabilidad social es un constructo usado para medir el grado en que una respuesta o un conjunto de respuestas de un individuo están siendo contestadas de modo discursivo, políticamente correcto o socialmente deseable y por ende, las respuestas dadas en las otras escalan no reflejarían las actitudes verdaderas si la correlación entre ambas es alta.

Las correlaciones de los indicadores con el indicador de Deseabilidad Social se calcularon en las pruebas piloto. El indicador que presentó mayor correlación fue el de "Actitudes hacia los roles de género", sin embargo esta correlación no fue lo suficientemente alta para descartar el indicador. Cuando la correlación por deseabilidad social es muy alta se debe eliminar la escala que representa el constructo y por eso es necesario hacer este análisis para cada una de las subdimensiones del CBV.

Otro tema pendiente es la unión de los tres componentes para medir empleabilidad, y el peso relativo de cada componente para predecir el acervo de empleabilidad de un adolescente particular. Dado que el indicador de empleabilidad se va usar para evidenciar resultados del programa, se necesita encontrar una variable externa que permita valorar la bondad de tal predicción. Este artículo representa un primer paso en esa dirección. Se espera, en un futuro cercano poder avanzar en los análisis pendientes. 


\section{REFERENCIAS}

Academy for Educational Development (2002). External Outcome Evaluation Plan. Documento contratado por la Agencia Patrocinadora del Proyecto. SE

Agencia Ejecutora (2003). Modelo Curricular de Oferta Interna. Documento de uso interno. SE.

Anastasi, A. \& Urbina, S. (1998). Tests Psicológicos. México: Prentice Hall.

Babbie, E. (2000). Fundamentos de la investigación social. International Thomson Editorial. Traducción del libro publicado en inglés por Wadsworth Publishing Company. 1999

Gable, R \& M. Wolf (1993). Instrument Development in the Affective Domain: measuring attitudes and values in corporate and school settings. Second Edition. Kluwer Academic Publishers. Fourth Printing. 2001

Jonhson, D. (1998). Métodos multivariados aplicados al análisis de datos. México.DF. Thomson Editores.

Nunnally, J.C. \& Bernstein, I.J. (1995). Teoría Psicométrica. México, D.F.: McGrawHill Interamericana. 